\title{
An Object Oriented Library for Sawmill Simulation
}

\author{
Francisco J. Ramis \\ Pablo Concha \\ Department of Industrial Engineering \\ University of Bio-Bio \\ Concepción, CHILE \\ Liliana Neriz \\ Department of Information Systems and Auditing \\ University of Chile \\ Santiago, CHILE \\ José A. Sepúlveda \\ Department of Industrial Engineering \\ and Management Systems \\ University of Central Florida \\ Orlando, Florida, USA.
}

\begin{abstract}
This work shows an object oriented library that was designed to facilitate the development of simulation studies of sawmills. A group technology approach was used to minimize the number of objects in the library, which includes objects that have the functionality of the equipments found in these facilities, so that the analyst only needs to provide the layout, locate the transports and machines, and insert the parameters of the process. For large sawmills having state of the art production equipment, with optimization capacities in each machine, two applications of the library are shown. The study demonstrates the advantage of using an object orientation, and the usefulness of simulation as a tool for aiding in both the design and in the improvement of the operations of these facilities.
\end{abstract}

Keywords: Object oriented simulation, Sawmill simulation, simulation library 


\section{Proceedings of the 51st International Convention of Society of Wood Science and Technology November 10-12, 2008 Concepción, CHILE}

\section{Introduction}

The paper presents an object oriented library, which was designed to facilitate the development of simulation studies of sawmills. A group technology approach was used to minimize the number of objects in the library, which includes objects that have the functionality of all the equipments found in these facilities, so that the analyst only needs to provide the layout, locate the transports and machines, and insert the parameters of the process. For large sawmills having state of the art production equipment with optimization capacities in each machine, two applications of the library are shown. The first one considers the improvement of an existing sawmill and the second one is an example where simulation is used to verify the operational parameters of a future sawmill. The study demonstrates the advantage of using an object orientation, and the usefulness of simulation is a tool for aiding in both the design and in the improvement of the operations of these facilities.

Many examples can be found in the literature where the use of simulation in the forest industry is reported. Brunner, Funck and Zhang (1994) present one of the earliest papers using object orientation for sawmill simulation. Wenjie, Kline, Araman and Wiedenbeck (1995) report on modeling different products through simulation. Baesler, Moraga and Ramis (2002) report on the use of simulation and artificial intelligence techniques to improve productivity in the wood industry; Ramis et Al. (2004) use simulation to identify and eliminate bottlenecks at a sawmill and Ramis, Palma and Concha (2005) use simulation to study the projected capacity of a sawmill. A current application of simulation is presented in Neriz et al., where goals are defined with the help of a simulation model to feed a Blance Scored Card of a sawmill plant. This paper takes full advantages of the object orientation paradigm to design a library to facilitate the model building of sawmill simulation studies.

\section{Sawmill Description}

As a background to understand the processes at a sawmill, a general description is given in this section. Modern sawmills are computerized facilities, where logs are loaded in one side and packages of lumber, ready to be sent to different clients, are obtained in the other side. In general terms, Figure 1 shows the process that follows the logs to become timber with the final dimensions for a particular market. The logs are loaded into a conveyor and then they go into positioning machine and a true shape scanner to determine the optimal way to produce the first cut at the first machine. The first cut is done with a single or multiple bandsaw machine with optimization capacities and a chipper canter. The first cut produces lateral wood, a semi-log and chips. The lateral wood, which is used for furniture and moldings, goes into the edgers machine to get a commercial width. The semi-log, which is used for packaging, goes into a second cut at a multisaws machine. The second cut produces additional lateral wood and a flitch from the central part of the log. The lateral wood goes into the edgers to give the final width to the lumber and the flitch goes into a third cut, for example at a multi rip gang machine, to produce lumber. At the end of 


\section{Proceedings of the 51st International Convention of Society of Wood Science and Technology November 10-12, 2008 Concepción, CHILE}

the model, conveyors take the lumber into a sorter and finally into a stacker to generate packages. The chips generated before each cut normally go into pulp mills.

From a schematic point of view, a sawmill is a collection of conveyors, which takes the logs or lumber through different machines until they get the final dimensions, as shown in figure 1.

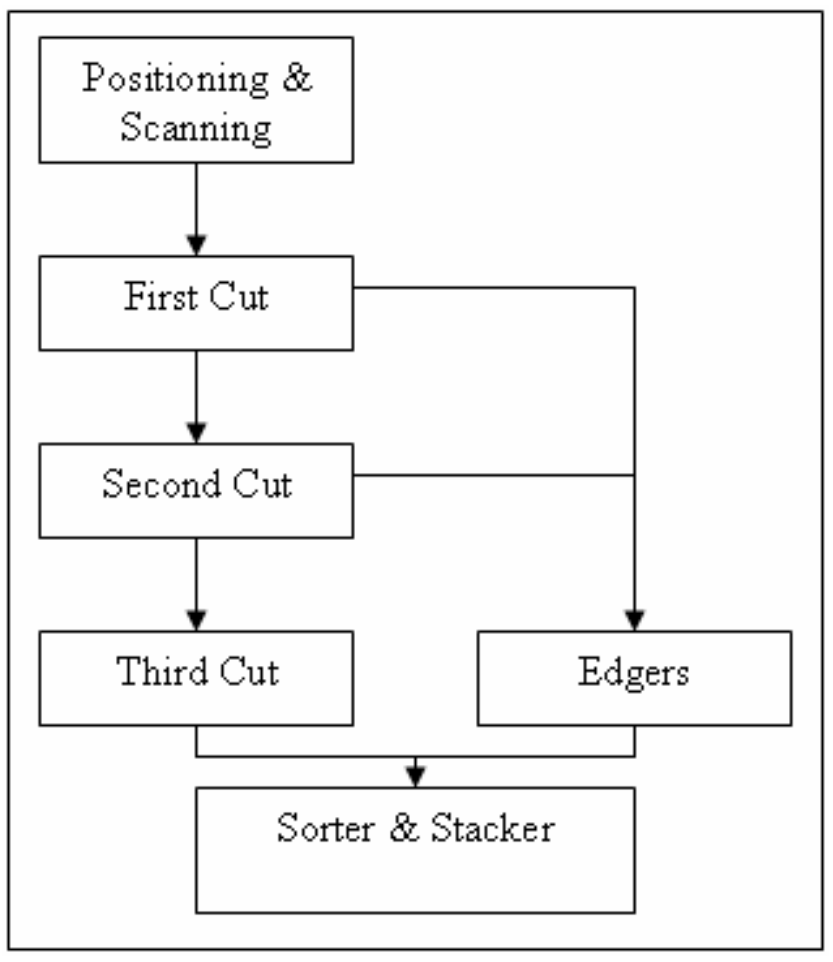

Figure 1. Process Flow

\section{Object Design Methology}

The hypothesis for designing a library for the sawmill industry was that by having a template, with all the machines and equipments that could be found in these kind of facilities, the model building could be achieved by dragging and dropping the equipments of the process, connecting them and setting the parameters in each object. An object oriented paradigm was selected for the flexibility it offers to build the objects with the required capacities and reusability.

Group technology was used to minimize the number of objects to be included, which was accomplished working with wood engineers, machine and equipment vendors, and process engineers. The minimum number of objects in the library was accomplished by iterating through the following sequence of questions: (1) What products are made at sawmills? (2) What different processes can be used to generate those products? and (3) What machines can be used in these processes? 


\section{Object Library}

As a result of the previous process, the object library shown in Figure 2 was built using Flexsim as the software development platform. With this library, having only 12 objects, almost any sawmill in the world may be represented. It includes a generator of logs, different types of sawing/cutting machines, a turn table, a queue, a feeder, and a conveyor. Each object was designed to include all the parameters and relevant capacities that the real machines have in practice (setup time, speed, number of saws, time between failures, time to repair, etc.), so that in order to build a model, the analyst only needs to provide the layout, drag and drop the machines and conveyors, connect them, input the parameters, and run the model.

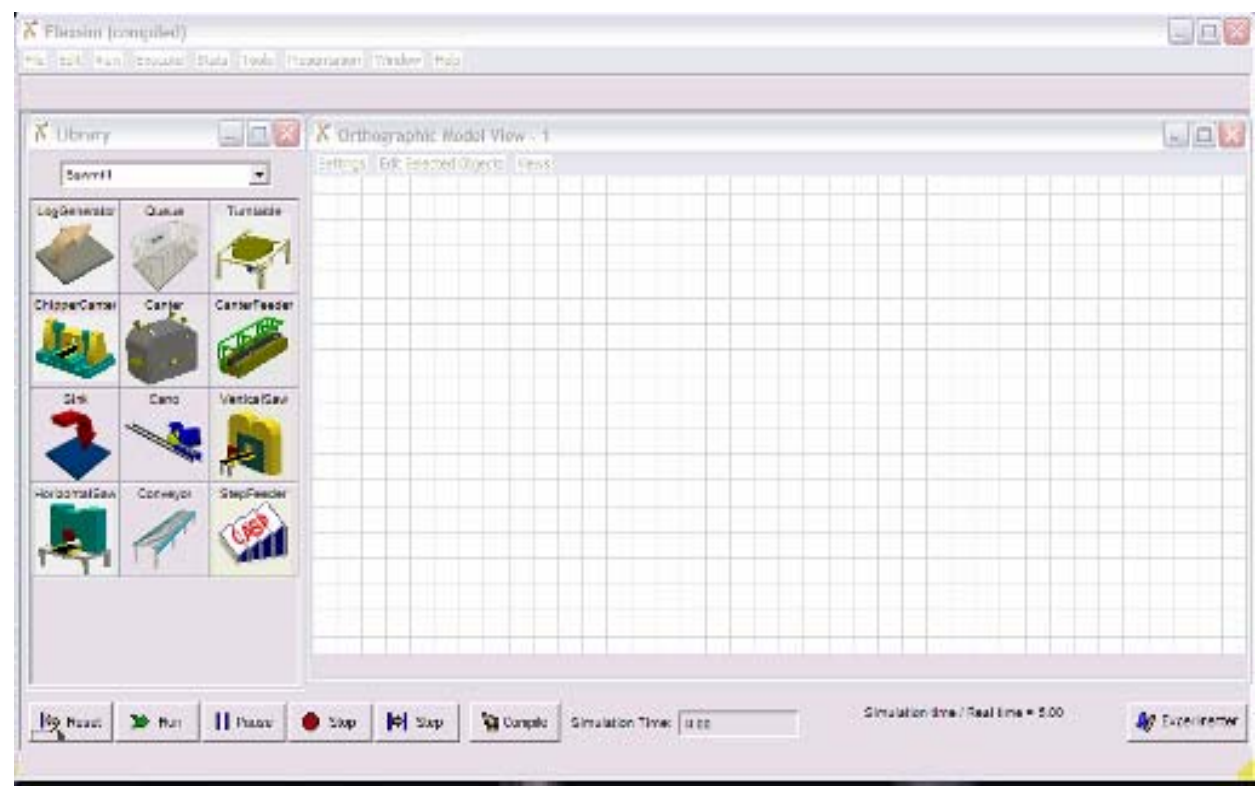

Figure 2. Object Library

Certainly, the object oriented library does not simplify the work of having to gather the input data and all the time consuming associated activities. In order to validate the library, different interviews and presentations where conducted with plant managers and production engineers at medium and large size sawmills. Also, simulation of different configurations of sawmills were done. Two of these experiments are reported here.

\section{Simulation of an Existing Sawmill}

The first example presented is an application of the library to an existing sawmill. The modeled system is an automated large sawmill having a production capacity of 20,000 cubic meters per month. It has machines with optimization capacities in the first and second cut, and edgers, with scanners previous to each machine. The simulation model 


\section{Proceedings of the 51st International Convention of Society of Wood Science and Technology November 10-12, 2008 Concepción, CHILE}

was designed to study the bottlenecks generated along the production line, and the model was oriented to analyze bottlenecks and evaluate improvement opportunities.

\section{Model Building}

In order to do the study, the methodology proposed by Law (2007) was used. The main objective of this study was to identify opportunities to improve the operation of the sawmill.

Data from the operation of the mill was gathered using databases and doing on-site data collection, which then was analyzed for inclusion in the model. The model was built using the customized library, with a significant reduction of time. In previous simulation of sawmills, the building of the computer model took at least a month of work and with the use of the library, this was reduced to about a day of work, including time to improve the presentation. The validation of the model was done comparing the simulation results with an actual production plan for the mill, plus the opinion of the production engineers, concluding that the simulation model was a good representation of the real system.

Bottlenecks were identified analyzing the use of the different machines in the process and by looking at the impact on total lumber production by increasing the processing speed of each machine. It was possible to identify that the main bottleneck is the machine performing the second cut (see figure 3), which also had the highest utilization factor as well as being responsible for stopping the system with the highest frequency. Sources of randomness are given by machine failures, log jams, and log diameter variability. The sawmill is fed with logs with a target diameter, but there exists variability on the distribution of diameters that arrives into the system. This issue is very important since the sawmill optimizes the wood utilization by positioning the saws at a different place as the diameters of the logs change, which generates setup time. In other words, if just one diameter were used, almost no setup times would be necessary.

The second bottleneck detected was at the edger machines. This resource becomes the bottleneck after solving the problem in the second cut. In order to analyze improvement opportunities in the system, a design of experiments study was conducted considering three main factors that may have an impact in the performance of these machines. The measure of effectiveness selected was the system's productivity (logs/month).

This information was used to construct a two-level full factorial experimental design. This type of design requires a total of $2 \mathrm{k}$ experiments, where $\mathrm{k}$ is the number of factors included in the analysis, which was fixed as 2. The factors and levels considered for analysis were:

- Diametric dispersion decreased. This factor considers a change in the classification of the logs fed to the mill. It was assumed that the base situation was that for any target diameter, $80.5 \%$ of the logs were on target and there was a deviation of plus/minus $10 \%$ in thee diameter. For example, if the target is to use $30 \mathrm{~cm}$. $\log , 80 \%$ will be in this class, 


\section{Proceedings of the 51st International Convention of Society of Wood Science and Technology November 10-12, 2008 Concepción, CHILE}

$10 \%$ above this diameter and $10 \%$ below. As an alternative, it was also simulated if the classification improves to $90 \%+/$ - from the target.

- Wood extractions. From simple inspection of the process, it was found that only around $50 \%$ of the wood needs to pass through the edgers to remove bark and get a different width. One alternative is to detour this wood using a bypass conveyor, which would reduce the load on the machines. The operation was simulated with and without extractions.

- Second cut speed increased. As a third factor, increasing the speed of the conveyor passing the semi-log for the second cut was considered. The base situation was the current speed, with the alternative increasing the speed by $10 \%$.

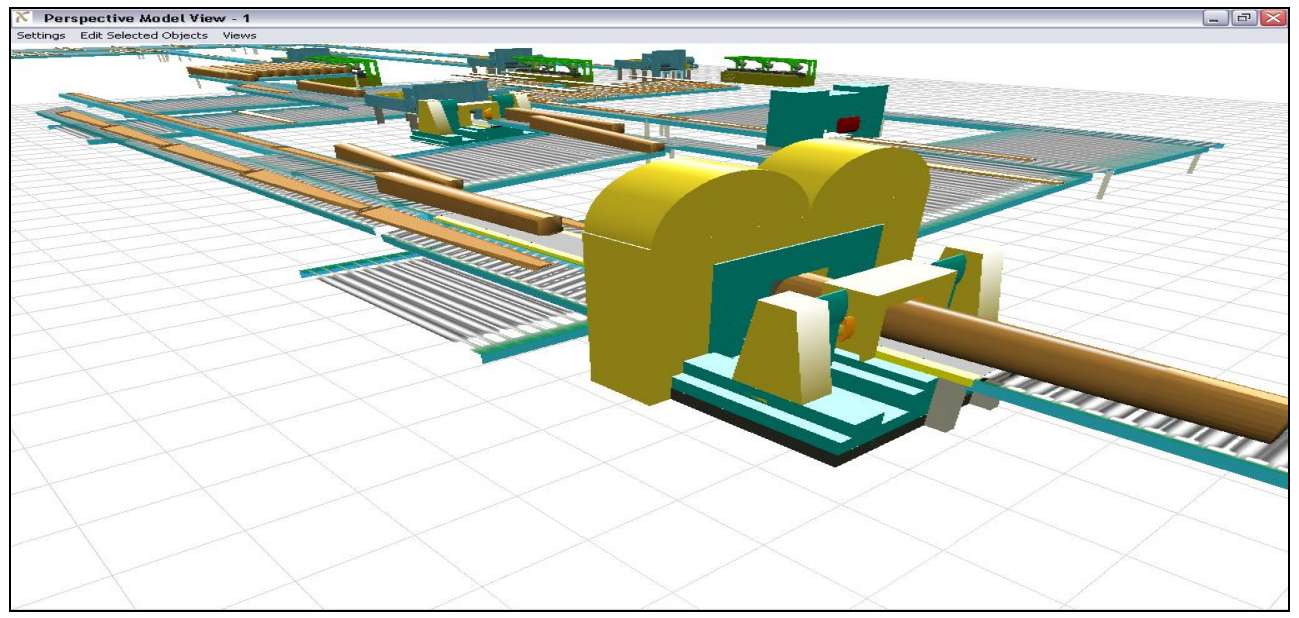

Figure 3. Sample Screen of existing sawmill

\section{Analysis of results}

The results from ten replicates for each factor and level allowed to conclude that only diameter dispersion and extractions were significant. An increased of the monthly production of logs can be seen when the dispersion is reduced to $+/ 5 \%$ from the target diameter. A pair t-test was conducted and concluded that the null hypothesis of no difference is rejected at a $90 \%$ confidence. By combining the effect of improved classification and extraction, the productivity of the mill is increased by $16.6 \%$, passing a total of 23,076 logs per month, compared to the base case of 19,795 logs.

\section{Simulation of a Future Sawmill}

This second example was an application of the library to a sawmill under design. The modeled system was a large sawmill having a projected production capacity of 30,000 cubic meters per month, which had state-of-the-art production technology. The simulation model was designed to verify operational parameters proposed by the vendor, in particular the piece count to be obtained with the machines and layout proposed, the 


\section{Proceedings of the 51st International Convention of Society of Wood Science and Technology November 10-12, 2008 Concepción, CHILE}

number of edgers required, and if a stacker with a capacity of 70 bins was enough to evacuate the products.

\section{Model Building}

As this was a projected facility, it was not possible to gather data from the actual system. The parameters to build the model were obtained from the vendor of the mill, the plant manager, and visiting other facilities with similar equipment. The verification process of the model was with a high level of detail to make sure that logs did not disappear in the process. Similarly, a face validation was performed with the vendor and plant manager.

In this model only deterministic data was employed. This is justified because the sequence of decisions is complex when considering the mix of products to be generated with different diameters of logs, two types of logs, and different cutting patterns. Three scenarios were designed to study the productivity of the mill (logs/month):

- Increasing number of edgers. This factor considers using three rather than the projected two edgers.

- Speed of the sawmill. Running the sawmill at the maximum velocity that the machines and conveyors can give, rather than the speed of the bottleneck machine.

- Using a different cutting pattern. This factor considers using an alternative cutting pattern to the base case projected.

\section{Analysis of results}

As a general conclusion of the experiment for this example is that the bottleneck moves from the central line for large diameters, to the edgers as the mill works with smaller diameters. Will small diameters the mill can run at a higher velocity and there is increased production of lateral wood, which congest the buffer conveyors before the edgers.

Specific conclusions are that the projected piece count will be achieved, and that the two edgers and the stacker are enough to achieve the desired production. It was verified that by adding a new edger, an increase in production of $22 \%$ could be achieved, which is contrasted with a projected $7 \%$ by the vendor of the mill.

\section{Conclusions}

This article presented an object oriented library for sawmills simulation built using Flexsim. The library was designed with the objective of simplifying the model building activities during a simulation study. The library was developed to include as objects all the elements needed to represent the machines and transport found in any sawmill. This library is applied to simulate two large sawmills having state-of-the-art production equipment, with optimization capacities in each machine. The first one considers the improvement of an existing sawmill, where an increase in productivity of $16.6 \%$ was 


\section{Proceedings of the 51st International Convention of Society of Wood Science and Technology November 10-12, 2008 Concepción, CHILE}

achieved. The second example is used to verify the operational parameters of a future sawmill. It is shown that the projected parameters will be achieved with the selected equipment and also that there is a significant opportunity for increasing production Finally, the experience shows that object oriented simulation is a useful tool to create customized and user friendly simulation environments, which by having a lower level of complexity, facilitates the building of sawmill simulation models.

\section{REFERENCES}

Brunner, Ch., J. Funck and G. Zhang, 1994. Discrete-event object-oriented modeling environment for sawmill simulation. Simulation, v 62, n 2, p. 119-130.

Baesler, F., M. Moraga and F. Ramis, 2002. Productivity improvement in the wood industry using Simulation and artificial intelligence, Proceedings Winter Simulation Conference, San Diego, USA.

Law, A., 2007. Simulation: Analysis and Modeling. John Wiley, N.Y.

Neriz, L, M.T. Bull y F. Ramis. 2005. Cuadro de Mando Integral Aplicado a la Gestión y Control de Proyecto de Innovación Tecnológica: Simulador de Plantas de Aserrío, ALTEC 2005, Salvador de Bahía, Brasil.

Ramis, F, R. Jarpa, J. Palma, J. M. Sánchez, F. Baesler and J. Sepúlveda, 2004. Bottleneck Analysis using simulation. American Institute of Industrial Engineering, Simulation Solutions 2004, Orlando.

Ramis, F., J. Palma and P. Concha. Analysis of Operational Parameters of a Projected Sawmill. International Academy of Wood Science, IAWS 2005, Concepcion.

Wenjie, L., D. Earl Kline, Ph. A. Araman and J. K. Wiedenbeck, 1995. Design and Evaluation of Log-to-Dimension Manufacturing Systems Using System Simulation. Forest Products Journal, 45(3):37-44. 